\title{
'|||||||||||||||||||||||||||||||||||||||||||||||||||||||||||||||||||.
}

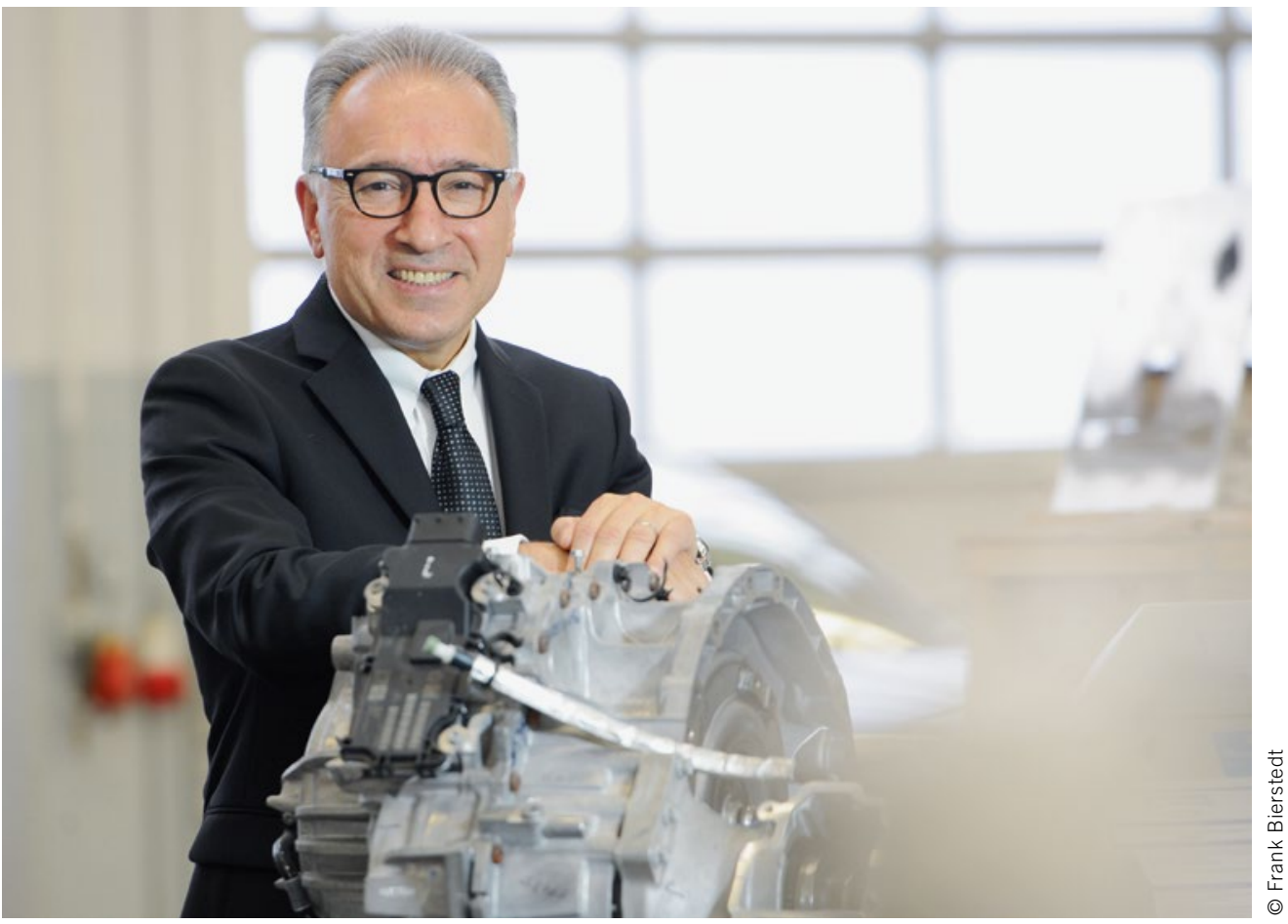

Prof. Dr.-Ing. Ferit Küçükay Direktor des Instituts für Fahrzeugtechnik der TU Braunschweig

\section{Hybridgetriebe im Vergleich}

Die weltweit am meisten verkauften Hybridgetriebe sind sogenannte Dedicated Hybrid Transmissions (DHTs) mit einem Anteil von $70 \%$, gefolgt von Add-on-Hybridgetrieben - hauptsächlich in P2-Konfiguration - mit $30 \%$. Einen Großteil der DHT machen die Powersplit-DHTs (PS-DHT) aus, bekannt durch den Toyota Prius. Den übrigen Anteil bilden die Multi-Mode-DHTs (MM-DHT), wie sie in den letzten Jahren beispielsweise durch den Voltec 2 von Chevrolet bekannt geworden sind.

Der Begriff DHT wurde als neue Konzeptklasse für Hybridgetriebe anlässlich der internationalen CTI-Symposia über Fahrzeuggetriebe, HEV- und EV-Antriebe in Berlin 2015 und in Detroit und Shanghai 2016 eingeführt und fand in der Fachwelt eine große Akzeptanz. Ein DHT beschreibt ein Getriebe, das neben der Verbrennungskraftmaschine (VKM) nur in Verbindung mit einer oder mehreren E-Maschinen funktioniert.

Bei Add-on-Konzepten für Hybridantriebe wird ein bestehendes Getriebekonzept mit einer E-Maschine erweitert, um Hybridfunktionen möglich zu machen. Damit sind Hybridantriebsstränge in P0- bis P4-Konfigurationen möglich. Diese sind bezüglich ihrer elektrischen Leistung skalierbar, mit 48-Voder Hochvoltbordnetz kombinierbar und dadurch in unterschiedlichen Fahrzeugsegmenten und Hybridisierungsgraden umsetzbar. Nachteilig ist bei einem P2-Hybrid der vergleichsweise schlechte mechanische Wirkungsgrad aufgrund der hohen Anzahl an Zahnrädern und Schaltelementen sowie der
Aktuatorik. Darüber hinaus erlaubt das Konzept als Parallelhybrid nur die Variation des VKM-Drehmoments durch die E-Maschine. Die Drehzahl ist durch die Gangübersetzung des Getriebes in jeder Fahrsituation fest vorgegeben.

In PS-DHTs und in MM-DHTs kann neben dem VKM-Drehmoment auch die VKM-Drehzahl variiert werden, was zu einer Betriebspunktoptimierung der VKM und somit einer höheren Antriebseffizienz führt. Dabei ist der mechanische Wirkungsgrad der MM-DHTs aufgrund der höheren Anzahl der reibungsbehafteten Bauteile etwas niedriger als bei den PS-DHTs. Bei Verwendung einer VKM mit identischer Leistung und gleichen Fahrleistungsanforderungen werden bei dem PS-DHT allerdings höhere E-Maschinen-Leistungen als beim MM-DHT und P2-Hybrid benötigt. MM-DHTs bieten mehrere Betriebsmodi. Dadurch ist es möglich - neben Hybridmodi - im rein elektrischen oder VKM-Betrieb mit mehreren Übersetzungen zu fahren, wodurch Fahrleistungs- und Effizienzvorteile gegenüber PS-DHTs entstehen. PS-DHTs und MM-DHTs haben allerdings gegenüber P2-Hybriden derzeit einen Kostennachteil, was in Zukunft durch höhere Produktionsvolumina und sinkende Kosten der elektrischen Komponenten kompensiert werden kann.

Im Zuge der zunehmenden Elektrifizierung der Antriebe und im Hinblick auf die relativ hohen erwarteten Marktanteile der Hybridantriebe stellen gerade die MM-DHTs eine interessante und zukunftsweisende Alternative dar. 